\title{
Banach Spaces and Classical Harmonic Analysis
}

\author{
S.V. Kislyakov
}

Vienna, Preprint ESI 711 (1999)

June 4, 1999

Supported by Federal Ministry of Science and Transport, Austria Available via http://www.esi.ac.at 


\title{
BANACH SPACES AND CLASSICAL HARMONIC ANALYSIS
}

\author{
S. V. Kislyakov
}

\begin{abstract}
Some points of contact of the two fields are discussed, specifically: projections onto translation invariant subspaces, Cohen's theorem and related results, multipliers of $H^{1}$, the use of invariant means, $p$-summing and $p$-integral operators, the vicinity of the Grothendieck theorem, some consequences of the Maurey-NikishinRosenthal factorization theorem, $\Lambda_{p}$-sets and Bourgain's solution of the $\Lambda_{p}$-problem, translation invariant subspaces without Gordon-Lewis local unconditional structure, Sidon sets, multipliers on spaces of vector-valued functions, specific spaces (related to harmonic analysis) in the general theory. Some proof are indicated or even exposed in detail, in case they are not technical and help to better illustrate the interplay between the fields in question.
\end{abstract}

Many classical Banach spaces admit a natural action of some group, and many specific operators commute with translations. This features can be used in the study of such spaces and operators. Reciprocally, sometimes the techniques of the Banach space theory apply in harmonic analysis.

This is nearly all that can be said about the subject of this paper in general. Furthermore, the manifestations of this relationship are scarce and heterogeneous. Invoking the polemical metaphor of Kahane and Salem for their classical book [KaS] on Fourier analysis ("il peut ... ressembler en quelque sorte à un herbier", [KaS, Préface]), it can probably be said that not only may the present text resemble somewhat, but it really is a "herbarium", much smaller and less systematized than [KaS], representing a far less explored taxon, and incomplete even in the known part of the latter.

In other words, the subsequent discussion can be viewed simply as a collection of examples. I hope, however, that the reader will find some intrinsic logic in them, and that at least sometimes he will be amused by the interplay of the two fields mentioned in the title.

1. Basic definitions. Throughout, "a group" means "an Abelian group". Any group $G$ acts on functions on it by translations $f \mapsto f_{x}$, where $f_{x}(y)=f(y+x)$, $x, y \in G$. A linear space $X$ of functions on $G$ is said to be translation invariant if with every function $f$ it contains all translates of $f$. Let $X$ and $Y$ be two translation invariant spaces on $G$, and let $T: X \rightarrow Y$ be a linear operator. Then $T$ commutes with translations if $T\left(f_{x}\right)=(T f)_{x}$ for $f \in X, x \in G$.

If $G$ is compact (which will be assumed in what follows unless otherwise is claimed explicitly), we denote by $d x$ the normalized Haar measure on $G$, by $\Gamma$ the dual group, and by $\hat{f}$ the Fourier transform of a function $f \in L^{1}(G)$ (the same notation is used for the Fourier transform of a measure). Mainly, we shall deal with

Supported in part by the Russian Foundation for Basic Research, Grant. no. 96-01-00693 
the classical spaces $C(G), L^{p}(G)(1 \leq p \leq \infty), M(G)=C(G)^{*}$. Let $X$ be any of these spaces, and let $E \subset \Gamma$. Then

$$
X_{E} \stackrel{\text { def }}{=}\{f \in X: \hat{f}(\gamma)=0 \text { for } \quad \gamma \in E\}
$$

is a translation invariant subspace of $X$. If $X=C(G)$ or $X=L^{p}(G)$ with $1 \leq$ $p<\infty$, all translation invariant subspaces are such. Next, the spaces $L_{E}^{\infty}(G)$ and $M_{E}(G)$ are the only $w^{*}$-closed translation invariant subspaces of $L^{\infty}(G)$ and $M(G)$, respectively.

In an obvious way, the symbol $X_{E}$ makes sense for other spaces $X$, and we shall use this notation without further explanations.

Now, let each of $X, Y$ be either $L^{p}, 1 \leq p<\infty$, or $C$, and let $E_{1}, E_{2} \subset \Gamma$. Suppose we are given a bounded linear operator $T: X_{E_{1}} \rightarrow Y_{E_{2}}$. Then $T$ commutes with translations if and only if it is representable in the form

$$
(T f)^{\wedge}=m \cdot \hat{f}
$$

where $m$ is a bounded function on $\Gamma$ vanishing on the complement of $E_{1} \cap E_{2}$ (note that, unless $X=Y=L^{2}$, not every bounded $m$ gives rise to a bounded operator via (1)). Often, $m$ is called the symbol of $T$. A similar characterization holds if the spaces $L^{\infty}$ or $M$ are involved, under the assumption of $w^{*}$-continuity of $T$.

We write $T=T_{m}$ if $T$ acts in accordance with (1); also, $T$ is called the multiplier with symbol $m$.

Among specific examples of translation invariant spaces, we mention the Hardy spaces $H^{p}\left(\mathbb{T}^{n}\right)$, where $\mathbb{T}^{n}$ is the $n$-dimensional torus. The dual group of $\mathbb{T}^{n}$ is $\mathbb{Z}^{n}$, and $H^{p}\left(\mathbb{T}^{n}\right)$ is simply the space $L_{\left(\mathbb{Z}_{+}\right)^{n}}^{p}\left(\mathbb{T}^{n}\right), 1 \leq p \leq \infty$. The space $C_{\left(\mathbb{Z}_{+}\right)^{n}}\left(\mathbb{T}^{n}\right)$ is called the polydisk algebra (the disk algebra if $n=1$ ). All these spaces have a well-known interpretation as the traces on the distinguished boundary $\mathbb{T}^{n}$ of certain spaces of functions holomorphic in the polydisk.

2. Averaging. Complementation. A most usual idea in the study of translation invariant subspaces is to average something. We start with simple and old examples; further, we shall come across several instances in which the realization of this idea is more intricate.

Again, let each of $X, Y$ be one of the spaces $L^{p}(G)(1 \leq p<\infty)$ or $C(G)$, and let $E_{1}, E_{2} \subset \Gamma$. For every bounded linear operator $T: X_{E_{1}} \rightarrow X_{E_{2}}$, we put

$$
\widetilde{T} f=\int_{G}\left(T f_{x}\right)_{-x} d x
$$

Then $\widetilde{T}$ commutes with translations. The mapping $T \mapsto \widetilde{T}$ is a norm 1 projection, and $\widetilde{T}$ can be referred to as the invariant part of $T$. Clearly, $\widetilde{T}$ inherits many properties of $T$, but it may happen that $\widetilde{T}=0$.

The oldest and most well-known applications in which $\widetilde{T}$ is quite substantial are related to projections. Suppose that $X=Y$ and $E_{2} \subset E_{1}$. If $P$ is a projection of $X_{E_{1}}$ onto $X_{E_{2}}$, it is easily seen that so is $\widetilde{P}$. The symbol $m$ of $\widetilde{P}$ is none other than the characteristic function of $E_{2}$.

In particular, it follows that the subspace $L_{E}^{p}(1 \leq p<\infty)$ (respectively, $C_{E}$ ) is complemented in $L^{p}$ (in $C$ ) if and only if the multiplier with the symbol $\chi_{E}$ is bounded on $L^{p}$ (on $C$ ). 
Proposition 1. For $1 \leq p<\infty, p \neq 2$, and $G$ compact infinite, some of the subspaces $L_{E}^{p}(G)$ are uncomplemented in $L^{p}(G)$.

Proof. By the preceding discussion, otherwise the characters of $G$ form an unconditional basis in $L^{p}(G)$. From the Khinchin inequality it is easy to deduce that this is not so if $p \neq 2$.

In the case of $L^{1}(G)$, much more can be said. We refer the reader to [Gr-McG] for the proof of the following statement.

Theorem 2 (Cohen's idempotent theorem). $L_{E}^{1}(G)$ is complemented in $L^{1}(G)$ if and only if $E$ is in the coset ring of $\Gamma$.

By definition, the coset ring of $\Gamma$ is the smallest system of sets containing all cosets of subgroups of $\Gamma$ and closed under finite unions and intersections and under complementation.

It is known that translation invariant operators of $L^{1}(G)$ or $C(G)$ into itself are precisely the operators of convolution with a finite measure. The projections among such operators correspond to the idempotent measures $\mu\left(\mu * \mu=\mu\right.$, or $\left.(\hat{\mu})^{2}=\hat{\mu}\right)$. So, Theorem 2 describes also the idempotent measures. Now, it is clear that the complemented translation invariant subspaces of $C(G)$ admit a characterization similar to Theorem 2.

A measure $\mu$ on $G$ is said to be quasiidempotent if $|\hat{\mu}|^{2} \geq|\hat{\mu}|$ (i.e., for each $\gamma \in \Gamma$ either $\hat{\mu}(\gamma)=0$ or $|\hat{\mu}(\gamma)| \geq 1)$. A set $E \subset \Gamma$ is called a quasi-Gordon set if there exists a quasiidempotent measure $\mu$ such that $E=\{\gamma: \mu(\gamma) \neq 0\}$. This notion was introduced in $[\mathrm{KwPe}]$ and will be used later on in this paper.

3. Invariant projections in $H^{1}(\mathbb{T})$. The subspace $H_{E}^{1}(\mathbb{T})$ may be complemented in $H^{1}(\mathbb{T})$ for some $E \subset \mathbb{Z}_{+}$not belonging to the Boolean ring generated by the arithmetic progressions and singletons (i.e., here the picture is different from that described in Theorem 2). The simplest example is $E=\left\{1,2,4, \ldots, 2^{n}, \ldots\right\}$.

Theorem 3 (Paley; see, e.g., $[\mathrm{H}, \mathrm{Z}]$ ). For $f \in H^{1}(\mathbb{T})$ we have

$$
\left(\sum_{n \geq 0}\left|\hat{f}\left(2^{n}\right)\right|^{2}\right)^{1 / 2} \leq c|| f \|_{1}
$$

A similar inequality is valid for any Hadamard lacunary set $E$ (we recall that $E \subset \mathbb{Z}_{+}$is said to be Hadamard lacunary if $E=\left\{m_{1}, m_{2}, \ldots\right\}$ with $m_{k+1} \geq \lambda m_{k}$ for a fixed constant $\lambda>1$ ). From the properties of lacunary trigonometric series, it follows that such an inequality means precisely that $T_{\chi_{E}}$ is a bounded operator on $H^{1}$.

Theorem 4 (see $[\mathrm{Kl}]$ ). The subspace $H_{E}^{1}(\mathbb{T})$ is complemented in $H^{1}(\mathbb{T})$ if and only if $E$ is in the Boolean ring of subsets of $\mathbb{Z}_{+}$generated by singletons, arithmetic progressions, and Hadamard lacunary sequences.

4. Now we discuss the complemented translation invariant subspace of $L^{1}(G)$ for $G$ locally compact but noncompact. H. P. Rosenthal showed (see [Ro]) that for such a space $X$ the set

$$
h X=\{\gamma \in \Gamma: \hat{f}(\gamma)=0 \text { for all } f \in X\}
$$

must be in the coset ring of $\Gamma_{d}$, i.e., of the group $\Gamma$ dual to $G$ and endowed with the discrete topology. This is, however, rather far from a complete description. We quote a final result for the group $\mathbb{R}$ (see $[\mathrm{AM}]$ ). 
Theorem 5. A translation invariant subspace $X$ is complemented in $L^{1}(\mathbb{R})$ if and only if $h X=\cup_{1<i<n}\left(\alpha_{i} \mathbb{Z}+\beta_{i}\right) \backslash F$, where $F \subset \mathbb{R}$ is a finite set, $\left\{x_{i}\right\}$ and $\left\{\beta_{i}\right\}$ are two finite collections of reals, and the numbers $\alpha_{i}$ are pairwise rationally independent.

The above general result of Rosenthal is again based on averaging, and we show how this procedure applies this time. Let $P$ be a projection onto a translation invariant subspace $X$ of $L^{1}(G)$. Then $P^{*}: X^{*} \rightarrow L^{\infty}(G)$ is a simultaneous extension operator, i.e., $P^{*}$ extends each bounded linear functional on $X$ to a bounded linear functional on $L^{1}(G)$ (and the latter is identified with a function in $L^{\infty}(G)$, as usual). Now, we define a simultaneous extension operator $\mathcal{E}: X^{*} \rightarrow L^{\infty}(G)$ commuting with translations as follows:

$$
\langle\mathcal{E} F, g\rangle=m_{x}\left\langle F,\left(P\left(g_{x}\right)\right)_{-x}\right\rangle, F \in X^{*}, g \in L^{1}(G) .
$$

Here $m$ is a fixed invariant mean on $l^{\infty}(G)$, i.e., a linear functional satisfying $m(1)=1, m \geq 0$, and $m\left(f_{x}\right)=m(f)$ for $f \in l^{\infty}(G), x \in G$ (the subscript $x$ in the notation $m_{x}$ shows that $m$ is applied "in the variable $x$ "). For $G$ commutative, such a functional always exists, see, e.g., [Gri].

Now, we see that the operator $A: F \mapsto F-\mathcal{E}\left(\left.F\right|_{X}\right)$ is a projection commuting with translations and taking $L^{1}(G)^{*}=L^{\infty}(G)$ onto $X^{-}=\left\{F \in L^{1}(G)^{*}:\left.F\right|_{X}=\right.$ $0\}$. Eventually, it turns out that Cohen's theorem can be applied to deduce Rosenthal's result, but some additional analysis is needed to do this. The crucial step is the observation that on the Bohr compactification $\widetilde{G}$ of $G$ we can find a measure $\mu$ such that $A f=f * \mu$ for every continuous almost periodic function $f$ on $G$. Since the dual group of $\widetilde{G}$ is precisely $G_{d}$, we easily arrive at Rosenthal's result with the help of Theorem 2. See [Ro] for more details.

5. Averaging against invariant means can also be used to show that, strikingly, certain statements involving the uniform structure of a Banach space can be linearized. Let $X$ be a Banach space, and $Y$ its closed subspace. Assume that there exists a linear operator $v$ mapping $Y^{*}$ to the space of uniformly continuous functions on $X$ such that $v f$ is an extension of $f$ for every $f \in Y^{*}$. Next, assume that $v$ is continuous for the topology of uniform convergence on bounded sets.

Theorem 6 (see [Pe2, Remarks to $§ 2$, Proposition A]). Under the above assumptions, there exists a bounded linear operator $w: Y^{*} \rightarrow X^{*}$ extending each $y^{*} \in Y^{*}$ to the entire space $X$.

An operator $v$ as above exists if, e.g., $Y$ is a uniform retract of $X$ (we may put $v f=f \circ p$ if $p: X \rightarrow Y$ is a uniform retraction). We infer that if, say, $Y$ is reflexive and uncomplemented in $X$, then $Y$ is not a uniform retract of $X$. In particular, no infinite dimensional reflexive subspace of $C(K)$ is a uniform retract of $C(K)$.

Proof of Theorem 6. The operator $w$ is given by the formula

$$
(w f)(z)=\int_{X} \int_{Y}[(v f)(x+y+z)-(v f)(x+y)] d y d x,
$$

where by $\int_{Y} \ldots d y$ and $\int_{X} \ldots d x$ we denote some fixed invariant means on $l^{\infty}(Y)$ and $l^{\infty}(X)$ ( $X$ and $Y$ are regarded as Abelian groups under their own operations of addition). The definition of $w$ is consistent because the "integrand" is bounded (note that, separately, the terms in the "integrand" are unbounded). That $w$ has the required properties is easy. The external "integration" is responsible for the additivity of $(w f)(\cdot)$, while the internal one for the relation $\left.(w f)\right|_{Y}=f$. 
6. $p$-summing operators. Let $G$ be a compact space, $X$ a subspace of $C(G)$, and $T: X \rightarrow Y$ a $p$-summing operator $(1 \leq p<\infty)$. By the Pietsch theorem (see "Basic Concepts"), the latter is equivalent to the existence of a probability measure $\mu$ on $G$ such that

$$
\|T f\| \leq c \int_{G}|f| d \mu, \quad f \in X .
$$

Proposition 7. If $G$ is a compact group, $X$ is translation invariant, and $T$ satisfies $\left\|T f_{x}\right\|=\|T f\|(f \in X, x \in G)$, then in (2) we can take the Haar measure of $G$ as $\mu$.

This is again done by averaging: we substitute $f_{x}$ for $f$ in (2) and integrate in $x$ against the Haar measure.

The condition on $T$ in Proposition 7 is fulfilled if, say, $Y=L^{r}(G)$ or $Y=C(G)$, and $T$ commutes with translations.

Proposition 8. Let $1 \leq p<\infty$, and let $E \subset \Gamma$. The identity embedding $i$ : $C_{E}(G) \rightarrow L_{E}^{p}(G)$ is p-integral if and only if the multiplier with symbol $\chi_{E}$ is bounded on $L^{p}(G)$.

Proof. The "if" part is clear. We prove the "only if" part in the case where $p>1$ (only slight complications arise for $p=1$, but we do not dwell on this). If $i$ is $p$-integral, we have the following factorization:

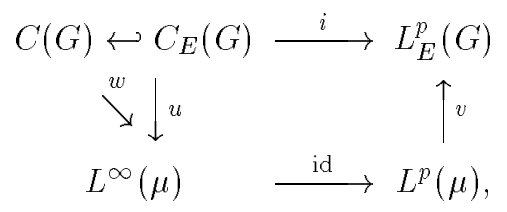

where $u, v$ are bounded linear operators, $\mu$ is a probability measure, and id is the identity inclusion. By the extension property of $L^{\infty}$, there exists an extension $w$ of $u$ making the diagram commutative. Thus, the operator $T=v$ id $w$ is a $p$-summing extension of $i$ to $C(G)$. It is easily seen that the averaged operator $\widetilde{T}$,

$$
\tilde{T} f=\int_{G}\left(T f_{x}\right)_{-x} d x
$$

is a $p$-summing extension of $i$ to $C(G)$ that commutes with translations. Now, Proposition 7 shows that $\widetilde{T}$ acts in fact from $L^{p}(G)$ to $L_{E}^{p}(G)$; consequently, the invariant projection of $L^{p}(G)$ onto $L_{E}^{p}(G)$ is bounded.

By Proposition 1 we now conclude that, if $G$ is infinite, some of the embeddings $C_{E}(G) \rightarrow L_{E}^{p}(G)$ are not $p$-integral (though, clearly, all of them are $p$-summing). This observation was made in [Pe1]. Trace duality then yields examples of quasi$p$-nuclear operators that are not $p$-nuclear.

7. Grothendieck theorem. This theorem says that any bounded linear operator $T: l^{1} \rightarrow l^{2}$ is 1 -summing. By the lifting property of $l^{1}$, to prove this it suffices to exhibit a single 1-summing operator onto $l^{2}$. Such an example can be provided by harmonic analysis (loosely, there is a multiplier with this property). This way to the Grothendieck theorem was indicated by Pełczyński and Wojtaszczyk around 1977 . 
Proposition 9. The operator $\tau: C_{\mathbb{C}_{+}}(\mathbb{T}) \rightarrow l^{2}$ given by $\tau f=\left\{\hat{f}\left(2^{n}\right)\right\}_{n \geq 1}$ is 1-summing and onto.

Proof. The fact that $\tau$ is 1 -summing is a consequence of Theorem 3. To show that $\tau$ is onto, it suffices to prove the estimate $\left\|\tau^{*} x\right\| \geq c\|x\|, x \in l^{2}$, which, by the F. and M. Riesz Theorem (see, e.g., [H]), is equivalent to the estimate

$$
\left\|\sum_{n \geq 1} x_{n} \bar{z}^{2^{n}}+p(z)\right\|_{L^{1}(\mathbb{T})} \geq c\left(\sum\left|x_{n}\right|^{2}\right)^{1 / 2}
$$

for every analytic polynomial $p$. We use the classical fact that the multiplier with symbol $\chi_{\mathbb{Z}_{-}}$maps $L^{1}(\mathbb{T})$ to $L^{r}(\mathbb{T})$ for every $r \in(0,1)$. This yields

$$
\left\|\sum_{n \geq 1} x_{n} \bar{z}^{2^{n}}+p(z)\right\|_{L^{1}(\mathbb{T})} \geq\left\|\sum_{n \geq 1} x_{n} \bar{z}^{2^{n}}\right\|_{L^{r}}
$$

It remains to refer to the classical properties of lacunary series.

Another curiosity of similar nature is the operator acting by the same formula as $\tau$, but defined on the space $C_{E}(\mathbb{T})$, where $E=\mathbb{Z}_{-} \cup\left\{2^{n}\right\}_{n} \geq 0$. It maps $C_{E}(\mathbb{T})$ onto $l^{2}$ and is $r$-summing for every $r>0$. This yields a direct proof (again via harmonic analysis) of Maurey's extension of the Grothendieck theorem: every operator from $l^{1}$ to $l^{2}$ is $r$-summing for every $r>0$. See the survey [Ki1] for the details.

8. Invariant Grothendieck theorem. We may state the Grothendieck theorem like this:

If $H$ is a Hilbert space, then for every operator $u: H \rightarrow C(K)$ the adjoint $u^{*}$ is 1 -summing.

The problem of replacing $C(K)$ in this statement by some other spaces has received considerable attention. In particular, it is reasonable to ask about the spaces of the form $C_{E}(G)$ that verify this theorem. We mention some cases in which this is indeed so.

(i) $\Gamma \backslash E$ is a $\Lambda_{p}$ set for some $p>1$ (equivalently, the space $L_{\Gamma \backslash E}^{1}$ is reflexive; $\Lambda_{p}$-sets will be discussed later in this article).

(ii) $G=\mathbb{T}, E=\mathbb{Z}_{+}$(then $C_{E}(G)$ is the disk algebra).

(iii) $G=\mathbb{T}^{n}, E=\mathbb{Z}^{n} \backslash\left(\mathbb{Z}_{-}\right)^{n}$.

(iv) $G=\mathbb{T}, E=\mathbb{Z}_{+} \cup\left\{-2^{n}\right\}_{n}>0$.

(v) $G=\mathbb{T}, E=\mathbb{Z}_{+} \cup\left(\bigcup_{k>0}\left[-2^{2 k+1},-2^{2 k}\right]\right)$.

Statement (i) is due to the author and Pisier; see, e.g., [DJT, Pi3] for the proof. More generally, if $Y$ is any reflexive subspace of $L^{1}(K, \mu)$, then the space $Y^{-}=$ $\left\{f \in C(K): \int g q u=0\right.$ for all $\left.g \in Y\right\}$ verifies the Grothendieck theorem, so that translation invariance is in fact irrelevant here. Statement (ii) is a celebrated result of Bourgain, the proof of which is based on fine Hardy space theory; see [GaKi, Ki1] for more information and references. Statement (iv) is easy modulo (ii), see the survey [Ki1]. Statements (iii) and (v) were proved, respectively, in [X] and [Ki2]. Curiously, the arguments are somewhat similar. They are based on the ideas of the proof of (ii), but involve some additional techniques (real variable Hardy spaces, Littlewood-Paley decompositions, etc.).

We see that, in spite of the (accidental) involvement of translation invariant subspaces, the methods leading to (i)-(v) can hardly be classified as deserving a 
more detailed exposition in this paper (at least, they deviate too much towards only one of the two items mentioned in the title, whereas the paper is about the vicinity of the conjunction "and").

The situation becomes different if we restrict ourselves to operators commuting with translations.

Definition (see $[\mathrm{KwPe}]$ ). A set $E \subset \Gamma$ is called a Marcinkiewicz set (respectively, a quasi-Marcinkiewicz set) if the multiplier with symbol $\chi_{E}$ is of weak type $(1,1)$ (respectively, there exists a bounded function $x$ on $\Gamma$ such a that $\alpha(\gamma)=0$ for $\gamma \notin E,|\alpha(\gamma)| \geq 1$ for $\gamma \in E$, and the multiplier with symbol $\alpha$ is of weak type $(1,1))$.

We remind the reader that an operator $T$ is of weak type $(1,1)$ if

$$
\operatorname{meas}\{|T g|>t\} \leq c t^{-1}\|g\|_{L^{1}}, \quad t>0
$$

for every $g$ in the domain of $T$.

Theorem 10 (see [KwPe]). If $E$ is a quasi-Marcinkiewicz set, then for every operator $u: L^{2}(G) \rightarrow C_{E}(G)$ commuting with translations the adjoint $u^{*}$ is 1 -summing.

The proof will be given in Section 10 .

It is well known that $\mathbb{Z}_{+}$is a Marcinkiewicz set for $G=\mathbb{T}$, but then Theorem 10 gives much less than statement (ii) above. The theory of martingale transformations yields many examples of Marcinkiewicz sets in the dual of the group $\prod_{n} \mathbb{Z}_{k_{n}}$ (see, e.g., [Gu]). Let, for instance, $k_{n}=2$ for all $n=1,2, \ldots$ (the dyadic group $D$ ). We agree that $\mathbb{Z}_{2}=\{-1,1\}$ (written multiplicatively). Let $\varepsilon_{j}$ be the $j$ th coordinate function of $D$; then all characters of $D$ except the function 1 are of the form $\varepsilon_{j_{1}} \varepsilon_{j_{2}} \ldots \varepsilon_{j_{N}}$, where $N=1,2, \ldots, j_{1}<j_{2}<\cdots<j_{N}$. Fixing an arbitrary set $B$ of integers, consider the set $E$ of the characters for which $j_{N} \in B$ in the above representation. Then $E$ is a quasi-Marcinkiewicz set.

9. It is interesting that Theorem 10 can be adapted to the space of smooth functions $C^{(k)}\left(\mathbb{T}^{l}\right)$. Neglecting the constant, we may endow this space with the norm

$$
\|f\|_{k}=\max _{|s|=k}\left|D^{s} f\right|
$$

equivalent to the standard one (as usual, $D^{s}$ denotes the parial derivative corresponding to a multiindex $s)$. Thus, in a natural way, $C^{(k)}\left(\mathbb{T}^{l}\right)$ can be regarded as a subspace $X$ of

$$
C\left(\mathbb{T}^{l}\right) \oplus \cdots \oplus C\left(\mathbb{T}^{l}\right)
$$

(the number of summands is equal to card $\{s:|s|=k\}$ ), and $X$ is invariant under simultaneous translation on all copies of $\mathbb{T}^{l}$. Moreover, the orthogonal projection onto the closure of $X$ in $L^{2}\left(\mathbb{T}^{l}\right) \oplus \cdots \oplus L^{2}\left(\mathbb{T}^{l}\right)$ is of weak type $(1,1)$.

In [KwPe] it was shown that, using this projection, it is possible to work with $X$ as if we had a space of the form $C_{E}(G)$ with a quasi-Marcinkiewicz set $E$. (The reader may trace this inspecting the proof in Section 10.) In particular, it follows that any operator $u: L^{2}\left(\mathbb{T}^{l}\right) \rightarrow C^{(k)}\left(\mathbb{T}^{l}\right)$ commuting with translations has 1 -summing adjoint. See $[\mathrm{KwPe}]$ for the details. 
10. Theorem 10 is proved by two-fold averaging. The first averaging yields a useful lemma stated below. Let each of $X$ and $Y$ be either $L_{E}^{p}(G)$ or $C_{E}(G)$ (maybe, with different $E$ 's), let $T: X \rightarrow Y$ be an operator commuting with translations, and let $S: Y \rightarrow X$ be a finite rank operator.

Lemma 11. We have trace $T S=$ trace $T \widetilde{S}$, where $\widetilde{S} f=\int_{G}\left(S f_{x}\right)_{-x} d x$.

Indeed, let $\sigma_{x}$ be the translation $f \mapsto f_{x}$. By the invariance of $T$ we have

$$
\operatorname{trace} T S=\operatorname{trace}\left(\sigma_{x}^{-1} T \sigma_{x} S\right)=\operatorname{trace}\left(T \sigma_{x} S \sigma_{x}^{-1}\right),
$$

and it suffices to integrate in $x$ over $G$.

Now, we remind the reader that the trace duality identifies the ideal of operators with 1-summing adjoint as the dual of the ideal $\Gamma_{1}$ of $L^{1}$-factorable operators; see [DJT]. In particular, for $u: L^{2}(G) \rightarrow C_{E}(G)$ we have

$$
\begin{gathered}
\pi_{1}\left(u^{*}\right)=\sup \left\{\operatorname{trace}(u v): v: C_{E}(G) \rightarrow L^{2}(G)\right. \text { is of } \\
\text { finite rank and } \left.\gamma_{1}(v) \leq 1\right\}
\end{gathered}
$$

Here, as usual, $\gamma_{1}$ stands for the norm on $\Gamma_{1}$. By Lemma 11, if $u$ commutes with translations, the supremum can be restricted to $v$ commuting with translations. We see that it suffices to estimate the nuclear norm $\nu_{1}(v)$ in terms of $\gamma_{1}(v)$ for any such $v: \nu_{1}(v) \leq c \gamma_{1}(v)$. We show a more general statement, without the finite rank assumption.

Theorem 12. If $E$ is a quasi-Marcinkiewicz set, then every 1-factorable operator $v: C_{E}(G) \rightarrow L^{2}(G)$ that commutes with translations is nuclear.

Proof. Again, we use an averaging procedure, but this time it is intricate. Let $T$ be a multiplier of weak type $(1,1)$ the symbol $\alpha$ of which satisfies $\alpha(\gamma)=0$ for $\gamma \notin E$ and $|\alpha(\gamma)| \geq 1$ for $\gamma \in E$. Clearly, $T$ maps $L^{1}(G)$ to $L^{1 / 2}(G)$. Next, since $v$ is $L^{1}$-factorable, from the Maurey extension of the Grothendieck theorem (see the end of Section 7 ) we deduce that $v$ is $1 / 2$-summing. Writing the definition of a $1 / 2$-summing operator in the integral form, we see that if $(\Omega, \lambda)$ is a measure space and $F: \Omega \rightarrow C_{E}(G)$ is a reasonably good function, then

$$
\int_{\Omega}\|v F(\omega)\|_{2}^{1 / 2} d \lambda(\omega) \leq \pi_{1 / 2}(v)^{1 / 2} \sup \left\{\int_{\Omega}|\langle F(\omega), \mu\rangle|^{1 / 2} d \lambda(\omega): \mu \in M(G),\|\mu\| \leq 1\right\}
$$

As $(\Omega, \lambda)$ we choose the group $G$ with the Haar measure, and we put $F(\omega)=$ $T\left(f_{\omega}\right), \omega \in G$, where $f$ is a trigonometric polynomial (a finite linear combination of characters) on $G$. Clearly, $F$ indeed maps $G$ to $C_{E}(G)$, and we obtain, using invariance:

$$
\|v T f\|_{2}^{1 / 2} \leq \pi_{1 / 2}(v)^{1 / 2} \sup \left\{\int_{G}\left|\left\langle T f_{\omega}, \mu\right\rangle\right|^{1 / 2} d \omega: \mu \in M(G),\|\mu\| \leq 1\right\} .
$$

Putting $\tilde{\mu}(e)=\mu(-e)$ and using the fact that $T$ maps $L^{1}$ to $L^{1 / 2}$, we see that

$$
\int_{G}\left|\left\langle T f_{\omega}, \mu\right\rangle\right|^{1 / 2} d \omega=\int_{G}|T(f * \tilde{\mu})|^{1 / 2} \leq C\left\|\left.f * \tilde{\mu}\right|_{1} ^{1 / 2} \leq C\right\| f \|_{1}^{1 / 2}
$$


It follows that $v T$ is a bounded operator from $L^{1}(G)$ to $L^{2}(G)$. Now, let $b$ be the symbol of $v$. We have

$$
\left(\sum_{\gamma \in E}|b(\gamma) \alpha(\gamma) \hat{f}(\gamma)|^{2}\right)^{1 / 2} \leq C \int_{G}|f|
$$

for every $f \in L^{1}(G)$. Letting $f$ run through some approximate identity, we infer that $\sum_{\gamma \in \Gamma}|b(\gamma)|^{2}<\infty$.

Thus, $v$ is the convolution with an $L^{2}$-function $V$ the spectrum of which is contained in $E$. So, $v$ factors as follows:

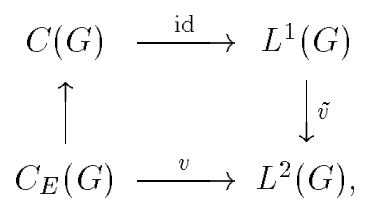

where $\tilde{v}$ is again convolution with $V$. Since id is 1-integral and $\tilde{v}$ maps to a reflexive space, $v$ is nuclear.

11. A counterexample. Returning to the beginning of Section 8 , let us agree to say that a space $X$ verifies the Grothendieck theorem if every operator from $l^{2}$ to $X$ has 1-summing adjoint. From the discussion in "Basic Concepts" (see [DJT] for more details), it can be seen that this happens if and only if every operator from $X$ to $l^{1}$ is 2-summing. From (ii) and (iv) in Section 8 we see that the spaces $C_{\mathbb{Z}_{+}}(\mathbb{T})$ and $C_{E}(\mathbb{T})$, where $E=\mathbb{Z}_{+} \cup\left\{-2^{n}\right\}_{n \geq 0}$, verify the Grothendieck theorem. However, their injective tensor product does not.

Indeed, this injective tensor pruduct is none other than $C_{\mathbb{Z}_{+} \times E}\left(\mathbb{T}^{2}\right)$, and the latter space contains a complemented copy of $l^{1}$ (then, surely, the projection to this copy is not 2-summing). This copy is spanned by the functions $\left\{z_{1}^{2^{n}} z_{2}^{-2^{n}}\right\}_{n}>0$. To exhibit a projection, we observe that the characteristic function of $\{(k,-k): k \in$ $\mathbb{Z}\} \subset \mathbb{Z}^{2}$ is the Fourier transform of a measure on $\mathbb{T} \times \mathbb{T}$. The required projection is given by convolution with this measure.

It is known that the dual spaces of $C_{\mathbb{Z}_{+}}$and $C_{E}$ are of cotype 2 (see, e.g., the survey [Ki1]). The above construction can be adapted to show that the projective tensor product of two cotype 2 spaces may fail to be of cotype 2, moreover, it may contain a complemented copy of $c_{0}$.

The conjectures disproved by the above were perceived as natural for some time in the past. A "more radical" answer to these and many other questions is given by Pisier's celebrated counterexamples (see, e.g., [Pi3]). I have included the above material (first published in [Ki1]) because of its transparence and relevance to harmonic analysis.

12. Stein theorem. Seemingly, in the proof of Theorem 12 we did not use the full-scale assumption that $E$ is a quasi-Marcinkiewicz set (we needed "only" the $\left(L^{1}-L^{1 / 2}\right)$ continuity of the corresponding multiplier). The result of Stein stated below shows that this is not so. We denote by $S(G)$ the space of all measurable functions on $G$ endowed with the (metrizable) topology of convergence in measure. 
Theorem 13. Let $\Lambda \subset \Gamma$, and let $T: L_{\Lambda}^{1}(G) \rightarrow S(G)$ be a continuous linear operator commuting with translations. Then $T$ is of weak type $(1,1)$.

Proof. The point is that something similar can be said if $T$ does not necessarily commute with translations. Specifically, by the Nikishin theorem (see [Ma]), in the latter case for every $\varepsilon>0$ we can find a set $\Omega_{\varepsilon} \subset G$ with $\left|G \backslash \Omega_{\varepsilon}\right|<\varepsilon$ such that

$$
\left|\left\{t \in \Omega_{\varepsilon}:|(T f)(t)|>\lambda\right\}\right| \leq\left. C_{\varepsilon} \lambda^{-1}|| f\right|_{1}, f \in L_{\lambda}^{1}, \lambda>0 .
$$

Now, if $T$ does commute with translations, it only remains to average. We fix, e.g., $\varepsilon=1 / 2$, and put $\Omega=\Omega_{1 / 2}$. Substituting $f_{x}$ for $f(x \in G)$ and using invariance, we rewrite (3) in the form

$$
\int \chi_{\Omega-x} \chi_{\{|T f|>\lambda\}} \leq C \lambda^{-1} \mid f \|_{1}
$$

integrating in $x$ over $G$, we get

$$
|\Omega||\{|T f|>\lambda\}| \leq C \lambda^{-1} \mid f \|_{1} .
$$

13. $\Lambda_{p}$-sets. Let $1 \leq p<\infty$. A subset $E$ of $\Gamma$ is called a $\Lambda_{p}$-set if in $L_{E}^{p}(G)$ norm convergence is equivalent to convergence in measure.

This is the same as if we demand the equivalence of the $L^{p}$ - and $L^{q}$-norms on $L_{E}^{p}$ for some $q<p$.

A subspace $X \subset L^{1}(\mu)$ (where $\mu$ is a finite measure) if reflexive if and only if the norm convergence on $X$ is equivalent to the convergence in measure; see [KadPe]. Thus, $E$ is a $\Lambda_{1}$-set if and only if $L_{E}^{1}(G)$ is reflexive.

The most well-known examples of $\Lambda_{p}$-sets (for all $p$ at once) are Hadamard lacunary sets of integers (here, of course, we have in mind the circle group $\mathbb{T}$ as $G$ ). In general, for a fixed $E$ the set $\left\{p: E\right.$ is of type $\left.\Lambda_{p}\right\}$ is an interval. In the pioneering paper [Ru] of Rudin it was shown that for any integer $n \geq 2$ there is a $\Lambda_{2 n}$-set not of type $\Lambda_{2 n+\varepsilon}$ for any $\varepsilon>0$. The main idea of the construction is that $\left|\sum_{\gamma \in E} a_{\gamma} \gamma\right|^{2 n}$ expands explicitly as a linear combination of products of the characters in $E$ and their complex conjugates, so that we may play with arithmetic conditions on $E$ to ensure huge cancellation after integration. This leads to nontrivial examples of $\Lambda_{2 n}$-sets, some of which turn out to be not of type $\Lambda_{2 n+\varepsilon}, \varepsilon>0$.

The question as to whether similar examples exist for $p \neq 2 n, n \geq 2, n \in \mathbb{Z}$, was often referred to as the $\Lambda_{p}$-problem.

For $p<2$, the solution of this problem has turned out to be easy (at least formally). Namely, if $p<2$, then every $\Lambda_{p}$-set is a $\Lambda_{p+\varepsilon}$-set for some $\varepsilon>0$. As in the preceding section, the reason is in the Rosenthal-Nikishin-Maurey factorization theorem saying, in particular, that if $X$ is any subspace of $L^{p}$ with $p<2$ on which the topologies of $L^{p}$ and $S$ are equivalent, then, after a change of density, $X$ becomes a subspace of $L^{r}$ with some $r>p$ :

$$
\left(\int\left|\frac{x}{a^{1 / p}}\right|^{r} a\right)^{1 / r} \leq C\left(\int\left|\frac{x}{a^{1 / p}}\right|^{p} a\right)^{1 / p}, x \in X
$$

where $a$ is a positive weight, $\int a=1$. See [Ma] for the details. Now, if $X$ is translation invariant, we easily get rid of the density by averaging, which implies 
our claim. This observation was made in [BE]. However (in reality, so-to-say), no specific examples of $\Lambda_{p}$-sets with $p<2$ that are not $\Lambda_{2}$-sets seem to be known (this is the precise meaning of the above remark on the formality of the solution in question).

For any $p>2$, Bourgain [Bo2] proved the existence of $\Lambda_{p}$-sets that are not of type $\Lambda_{p+\varepsilon}$. (Thus, the $\Lambda_{p}$-problem remains unresolved for $p=2$ only.) Bourgain's proof is probabilistic and combinatorial in nature. Technically, it has little in common either with harmonic analysis or with Banach space theory, though philosophically the result may be linked with the latter.

It is a known fact of the finite-dimensional theory of Banach spaces that if $X$ is a space of dimension $n$ and its cotype $p$ constant is at most $C$, then a typical subspace of $X$ of dimension $\left[C_{1} n^{2 / p}\right]$ is 2-distant from the Hilbert space of the same dimension. Here $C_{1}$ depends only on $C$. See [MiSch, Theorem 9.6]. Thus, in any $n$-dimensional subspace $X$ of $L^{p}(p>2)$ there are many nearly Hilbertian subspaces of dimension proportional to $n^{2 / p}$. Now, suppose that $X$ is spanned by characters $\gamma_{1}, \ldots, \gamma_{n}$. If we succeed in finding a subspace $Y$ of $X$ also spanned by characters, of dimension roughly $n^{2 / p}$, and such that the $L^{p}$-norm is equivalent to the $L^{2}$-norm on $Y$ with a constant independent of $X$, we are very close to the desired example. (In other words, we need to know that subspaces with "typical" behavior occur even among quite specific spaces, namely, among those spanned by characters.)

To understand why this finite-dimensional statement suffices, we note that in many cases the exponent $2 / p$ in the above discussion is optimal. If we restrict ourselves only to the spaces $X$ having this property, then on the above $Y$ the best constant of equivalence of the $L^{p}$ - and $L^{p+\varepsilon}$-norms must tend to infinity as $n \rightarrow \infty$, for every $\varepsilon>0$. Then we attach such spaces $Y$ to one another to obtain an infinite set that is precisely of type $\Lambda_{p}$.

In [Bo2] an infinite set of this sort was constructed by considering the spaces span $\left\{z^{2^{n}}, \ldots, z^{2^{n+1}}\right\}$ on $\mathbb{T}$ in the role of $X$; the "attachment" procedure consisted in applying the Littlewood-Paley decomposition. This is easy. It is the above finite-dimensional statement that is really difficult. Bourgain showed that, in fact, translation invariance is irrelevant in it.

Theorem 14 (see [Bo2]). Let $\varphi_{1}, \ldots, \varphi_{n}$ be mutually orthogonal functions on a probability space $(\Omega, \nu)$, let $\left|\varphi_{i}\right| \leq 1$ for all $i$, and let $2<p<\infty$. Then there is a subset $S \subset\{1, \ldots, n\}$ of cardinality at least $n^{2 / p}$ satisfying

$$
\left\|\sum_{i \in S} a_{i} \varphi_{i}\right\|_{p} \leq C(p)\left(\sum_{i \in S}\left|a_{i}\right|^{2}\right)^{1 / 2}
$$

for all scalar sequences $\left\{a_{i}\right\}$. The constant $C(p)$ depends only on $p$.

In fact, in a due probability sense, most subspaces $S$ of $\{1, \ldots, n\}$ possess the required property.

Later, Talagrand gave a different proof of this remarkable theorem (see [T]). Talagrand's proof is somewhat simpler than Bourgain's, but it leans upon finer probabilistic background. Also, there is some more philosophy of Banach space geometric nature around Talagrand's proof. We quote an intermediate statement occurring in [T]. In the case of $X=L^{p}$ it is weaker than Theorem 14; however, it is applicable in a more general setting. 
The norm of a Banach space $X$ is said to be 2-smooth if $\|x+y\|+\mid x-y \| \leq$ $2+C\|y\|^{2}$ with $C$ independent of $x$ and $y$. It is known that for $p \geq 2$ the norm of $L^{p}$ is 2-smooth.

Now, let $\left\{x_{i}\right\}_{1 \leq i \leq n}$ be a collection of vectors in a real Banach space $X$, and let

$$
\tau=\sup \left\{\sum_{1 \leq i \leq n} f\left(x_{i}\right)^{2}: f \in X^{*},\|f\| \leq 1\right\} .
$$

Talagrand showed that if the norm of $X$ is 2-smooth and $m=\left[n^{1-\varepsilon} / \tau\right](\varepsilon \in(0,1)$ is an arbitrary fixed number), then there exists a subset $I$ of cardinality $m$ in $\{1, \ldots, n\}$ such that

$$
\left.\left\|\sum_{i \in I} a_{i} x_{i}\right\| \leq K^{\prime} \sum_{i \in I}\left|a_{i}\right|^{2}\right)^{1 / 2} .
$$

(In fact, "most" subsets $I$ of cardinality $m$ are such.) Here $K$ depends only on $\varepsilon$ and on the constant $C$ in the definition of 2-smoothness.

We observe that if $X=L^{p}$, then $\tau \leq n^{1-2 / p}$, so that the statement is " $\varepsilon$-close" to Theorem 14. The reader is referred to [T] for the proof of the above result, and also to the procedure of eliminating $\varepsilon$ in the case of the $L^{p}$-norm, $p>2$. By the way, the method generalizes to some other norms (an analog of Theorem 14 for the Lorentz space $L^{p, 1}$ was presented in [T]). We do not have the possibility of entering into the technicalities of either Bourgain's or Talagrand's proof.

14. Sidon sets. A subset $E$ of $\Gamma$ is called a Sidon set if $\sum_{\gamma \in E}|\hat{f}(\gamma)| \leq c \mid f \|_{\infty}$ for every $f \in L_{E}^{\infty}(G)$. The smallest constant $c$ is called the Sidon constant of $E$ and is denoted by $S(E)$. Again, the most well-known example is given by the Hadamard lacunary sequences (for $G=\mathbb{T}$ ). Also, the coordinate functions of the dyadic group (viewed as characters) constitute a Sidon set. Basically, the latter sequence is none other than the sequence of Rademacher functions on the segment $[0,1]$.

It is well known that every Sidon set $E$ is a $\Lambda_{p}$ set for all $p \in[1, \infty)$ and, moreover,

$$
\|f\|_{p} \leq c \sqrt{p}|| f \|_{2} \quad \text { for } \quad f \in L_{E}^{2}(G), \quad p \geq 1
$$

see, e.g., [LoR]. A deep result of Pisier [Pi1] shows that, in fact, (4) is a characterization of Sidon sets.

We shall discuss Sidon sets in more detail later on. Here we mention only a recent result of Kalton and Pełczyński [KalPe] who showed that if $E \subset \Gamma$ is a Sidon set, then $L_{\Gamma \backslash E}^{1}(G)$ is not an $\mathcal{L}^{1}$-space (the question about this had been circulating for some time before that). In fact, again, the group structure has turned out to be irrelevant here.

Theorem 15. If $\mu$ is a finite measure and $Q$ is a surjection of $L^{1}(\mu)$ onto a space containing a copy of $c_{0}$, then $X=\operatorname{Ker} Q$ is not complemented in $X^{* *}$ and the Grothendieck theorem fails for $X$ (in the sense that there is an operator from $X$ to $l^{2}$ that is not 1-summing). Consequently, $X$ is not an $\mathcal{L}^{1}$-space and is not isomorphic to a Banach lattice.

See $[\mathrm{KalPe}]$ for the proof and related results. 
15. Gordon-Lewis local unconditional structure. We refer the reader to "Basic Concepts" for the precise definition, recalling only that a Banach space $X$ possesses Gordon-Lewis local unconditional structure (G-L l.u.st.) if and only if $X^{*}$ is a complemented subspace of a Banach lattice. Harmonic analysis provides many examples of spaces without local unconditional structure.

Theorem 16 (Kwapień and Pelczyński [KwPe]). If $E$ is a quasi-Marcinkiewicz set and there is a bounded function $\varphi \notin l^{2}(E)$ such that the multiplier $T_{\varphi}$ maps $L_{E}^{1}$ to $L_{E}^{2}$, then $C_{E}(G)$ fails to have $G-L$ l.u.st.

Theorem 17 (Pisier). If $E$ is a $\Lambda_{2}$-set, then $E$ is a Sidon set if and only if $C_{E}(G)$ has G-L l.u.st.

Theorem 18 (Pisier). Let $E$ be a $\Lambda_{2}$-set, and let $p>2$. Then $E$ is a $\Lambda_{p}$-set if and only if $L_{E}^{p}(G)$ has $G$-L l.u.st.

Remark. The "only if" parts of Theorems 17 and 18 are trivial because $C_{E} \sim l^{1}(E)$ for every Sidon set and $L_{E}^{p} \sim l^{2}(E)$ for every $\Lambda_{p}$-set if $p>2$.

The proofs will be given in the next section, along the lines of the paper [KwPe]. Here we make some comments. In Theorem 16 , let $G=\mathbb{T}, E=\mathbb{Z}_{+}$. Then $E$ is a Marcinkiewicz set. As $\varphi$, we may take either $\chi_{\left\{2^{n}: n \geq 0\right\}}$ (see Theorem 3), or the function $n \mapsto(n+1)^{-1 / 2}, n \in \mathbb{Z}_{+}$(then the continuity of $T_{\varphi}: H^{1} \rightarrow H^{2}$ is a consequence of the classical Hardy inequality $\sum_{n \geq 0}(n+1)^{-1}|\hat{f}(n)| \leq c|| f \|_{1}$, $f \in H^{1}$; see $\left.[\mathrm{H}]\right)$. So, we recover the well-known fact that the disk algebra fails to have G-L l.u.st.

Also (compare with Section 9), the proof of Theorem 16 can be adapted to show that the space $C^{(l)}\left(\mathbb{T}^{k}\right)$ does not have G-L l.u.st. if $k \geq 2$ and $l \geq 1$. Clearly, it suffices to prove this only for $l=1, k=2$. Then the role of $T_{\varphi}$ in Theorem 16 can be played by the Sobolev embedding operator $W_{1}^{(1)}\left(\mathbb{T}^{2}\right) \hookrightarrow L_{2}\left(\mathbb{T}^{2}\right)$. In Section 9 it was already explained that $C^{(1)}\left(\mathbb{T}^{2}\right)$ is similar to a space of the form $C_{E}(G)$ with $E$ a quasi-Marcinkiewicz set. The adjustment of the details is left to the reader (or see $[\mathrm{KwPe}])$.

Passing to applications of Theorem 17, we take $E=\left\{2^{n}: n \geq 0\right\} \subset \mathbb{Z}$ (this is a Sidon set for $\mathbb{T}$ ) and consider its square

$$
E_{1}=\left\{\left(2^{k}, 2^{l}\right): k, l \geq 0\right\} \subset \mathbb{Z}^{2} .
$$

From condition (4) for $E$ it is easily seen that $E_{1}$ is a $\Lambda_{p}$-set for $\mathbb{T}^{2}$ for every $p<\infty$. However, it is also easy to observe that in the analog of (4) for $E_{1}$ the constant grows as $p$, i.e., faster than $\sqrt{p}$. Thus, $E_{1}$ is not a Sidon set. By Theorem 17, $C_{E_{1}}\left(\mathbb{T}^{2}\right)$ fails to have G-L l.u.st. (we note that $C_{E_{1}}\left(\mathbb{T}^{2}\right)$ is isomorphic to the projective tensor product of $l^{1}$ by itself).

The domain of applicability of Theorem 18 is outlined in Section 13.

We refer the reader to $[\mathrm{KwPe}]$ for more examples.

16. Theorems 16, 17, and 18 are proved by similar methods. For Theorem 16, we consider the following operators:

$$
C_{E}(G) \stackrel{\text { id }}{\rightarrow} L_{E}^{1}(G) \stackrel{T_{\varphi}}{\rightarrow} L_{E}^{2}(G) \stackrel{T_{\psi}}{\longrightarrow} C_{E}(G) .
$$

Here $\psi$ is an arbitrary function in $l^{2}(E)$. By Theorem 12 , the adjoint $T_{\psi}^{*}$ is 1 summing. If $C_{E}(G)$ has G-L l.u.st., the identity embedding id factors through $L^{1}$ 
(because id is 1-summing; see [DJT]). So, we have come across a composition of operators belonging to mutually adjoint operator ideals, hence it follows that

$$
\nu_{1}\left(T_{\varphi} \operatorname{id} T_{\psi}\right) \leq c\left\|T_{\varphi}\right\|\|\psi\|_{l^{2}(E)}
$$

( $\nu_{1}$ stands for the nuclear norm), or

$$
\sum_{\gamma \in E}|\psi(\gamma)||\varphi(\gamma)| \leq C^{\prime}|| \psi \|_{l^{2}(E)}
$$

This contradicts the condition $\varphi \notin l^{2}(E)$.

For Theorem 17, we consider the following operators:

$$
C_{E}(G) \stackrel{\text { id }}{\rightarrow} L_{E}^{1}(G) \stackrel{k}{\rightarrow} L_{E}^{2}(G) \stackrel{T}{\rightarrow} C_{E}(G) .
$$

Here $k$ is the formal identity; $k$ is continuous because $E$ is a $\Lambda_{2}$-set. Next, $T$ is the convolution with a function $x \in C_{E}(G)$. We claim that $T^{*}$ is 1 -summing. Indeed, $T^{*}$ is an operator of convolution with $x(-t)$; convolving this function with a measure, we obtain a function in $C_{-E}(G)$, which does not depend on a particular choice of this measure in a fixed coset modulo $C_{E}(G)^{-}$. Hence, $T^{*}$ acts as follows:

$$
T^{*}: C_{E}(G)^{*} \stackrel{(\cdot) * x(-t)}{\longrightarrow} C_{-E}(G) \stackrel{\mathrm{id}_{1}}{\longrightarrow} L_{-E}^{1}(G) \stackrel{k_{1}}{\longrightarrow} L_{-E}^{2}(G),
$$

where, as in (5), $\operatorname{id}_{1}$ is the identity embedding and $k_{1}$ is a formal identity; thus, $T^{*}$ is 1 -summing.

Now, in (5) id is 1-summing, so that, if $C_{E}(G)$ has G-L l.u.st., as in the preceding proof we obtain

or

$$
\nu_{1}(k \text { id } T) \leq C\|x\|_{\infty}, \quad x \in C_{E}(G)
$$

$$
\sum_{\gamma \in E}|\hat{x}(\gamma)| \leq C\|x\|_{\infty}, \quad x \in C_{E}(G)
$$

Thus, $E$ is a Sidon set.

For Theorem 18, we let $q^{-1}+p^{-1}=1$ and $f \in L^{q}(G)$. Then, regarded as a mapping from $L_{E}^{p}(G)$ to $L_{E}^{2}(G)$, the operator $S$ of convolution with $f$ factors as follows:

$$
S: L_{E}^{p}(G) \stackrel{\tau}{\rightarrow} L_{E}^{\infty}(G) \stackrel{\text { id }}{\rightarrow} L_{E}^{1}(G) \stackrel{k}{\rightarrow} L_{E}^{2}(G) .
$$

Here $\tau$ is again the operator of convolution with $f$, and $k$ is the formal identity.

We see that $S$ is 1-summing. If $L_{E}^{p}(G)$ has G-L l.u.st., then $S$ factors through $L^{1}(\nu)$ for some measure $\nu$. Next, for $p>2$ every operator from a subspace of $L^{p}$ to $L^{1}(\nu)$ factors through a Hilbert space $H$ (see [Pi3, Chapter 3]). This implies the following factorization for $S$ :

$$
S: L_{E}^{p}(G) \stackrel{u}{\rightarrow} H \stackrel{v}{\rightarrow} L^{1}(\nu) \stackrel{u}{\rightarrow} L_{E}^{2}(G) .
$$

By the Grothendieck theorem, $w$ is 1-summing. Hence, $w v$ is Hilbert-Schmidt, with Hilbert-Schmidt norm not exceeding $C\|f\|_{q}$. Since $(w v)^{*}$ is also Hilbert-Schmidt with the same norm, it follows that

$$
\left(\sum_{\gamma \in-E}\left\|S^{*}(\gamma)\right\|^{2}\right)^{1 / 2} \leq C\|f\|_{q}, \quad f \in L^{q}(G),
$$

or, equivalently,

$$
\left(\sum_{\gamma \in E}|\hat{f}(\gamma)|^{2}\right)^{1 / 2} \leq C|| f \|_{q}, \quad f \in L^{q}(G) .
$$

A simple duality argument shows that the latter condition is equivalent to the fact that $E$ is a $\Lambda_{p}$-set. 
17. Quasi-Cohen sets vs quasi-Marcinkiewicz sets. It turns out that in Theorem 16 the condition of the existence of $\varphi$ admits a nice reformulation. See the end of Section 2 for the definition of a quasi-Cohen set.

Theorem 19 (Kwapień and Pelczyński, [KwPe]). The following properties of a set $E \subset \Gamma$ are equivalent.

(i) E is a quasi-Cohen set.

(ii) For every multiplier $T_{\varphi}: L_{E}^{1}(G) \rightarrow L_{E}^{2}(G)$ we have $\varphi \in l^{2}(E)$.

(iii) There is a constant $K$ such that for every trigonometric polynomial $p$ satisfying $\hat{p}(\gamma) \geq 0$ for $\gamma \in E$ we have $\sum_{\gamma \in E} \hat{p}(\gamma) \leq K\|p\|_{\infty}$.

Proof. (i) $\Rightarrow$ (ii). Let $\mu$ be a measure satisfying $\hat{\mu}(\gamma)=0$ for $\gamma \notin E,|\hat{\mu}(\gamma)| \geq 1$ for $\gamma \in E$, and let $S$ be the operator of convolution with $\mu$. Then $T_{\varphi} S$ maps $L^{1}(G)$ to $L_{E}^{2}(G)$, i.e.,

$$
\left(\sum_{\gamma \in E}|\hat{\mu}(\gamma) \varphi(\gamma) \hat{f}(\gamma)|^{2}\right)^{1 / 2} \leq c \int|f|, \quad f \in L^{1}(G)
$$

Letting $f$ run through some approximate identity, we infer that

$$
c \geq\left(\sum_{\gamma \in E}|\hat{\mu}(\gamma)|^{2}|\varphi(\gamma)|^{2}\right)^{1 / 2} \geq\left(\sum_{\gamma \in E}|\varphi(\gamma)|^{2}\right)^{1 / 2} .
$$

(ii) $\Rightarrow$ (iii). Let $\hat{p}(\gamma) \geq 0$ for $\gamma \in E$. We introduce the multiplier $T \sqrt{\hat{p} \mid E}$ : $L_{E}^{1}(G) \rightarrow L_{E}^{2}(G)$. To estimate the norm of this operator, for $f \in L_{E}^{1}$ we write

$$
\sum_{\gamma \in E}(\sqrt{\hat{p}(\gamma)}|\hat{f}(\gamma)|)^{2}=\int_{G} \overline{f(-x)}(p * f)(x) d x \leq\|f\|_{1}\|f * p\|_{\infty} \leq\|f\|_{1}^{2}|| p \|_{\infty},
$$

hence $\|T \sqrt{\hat{p} \mid E}\| \leq\|p\|_{\infty}^{1 / 2}$. From (ii) we deduce that $\sum_{\gamma \in E} p(\gamma) \leq C\|p\|_{\infty}$.

(iii) $\Rightarrow($ i). In the space $C(G)$, we introduce two convex set:

$$
\begin{gathered}
W=\left\{p \in C(G): \hat{p}(\gamma) \geq 0 \quad \text { for } \quad \gamma \in E, \quad \sum_{\gamma \in E} \hat{p}(\gamma)=K\right\} \\
U=\left\{q_{1}+q_{2}:\left\|q_{1}\right\|_{\infty}<1, \quad \hat{q}_{2}(\gamma) \leq 0 \quad \text { for } \quad \gamma \in E\right\}
\end{gathered}
$$

Here $K$ is the constant occurring in statement (iii).

Clearly, $W$ is closed and $U$ is open. Also, $W \cap U=\varnothing$. Indeed, if $p \in W$ and $p=q_{1}+q_{2}$ as in the definition of $U$, then $\left.\left(\hat{p}-\hat{q}_{2}\right)\right|_{E} \geq 0$ and

$$
K=\sum_{\gamma \in E} \hat{p}(\gamma) \leq \sum_{\gamma \in E}\left(\hat{p}(\gamma)-\hat{q}_{2}(\gamma)\right) \leq K \mid p-q_{2}\left\|_{\infty}=K\right\| q_{1} \|_{\infty}<K .
$$

Thus, there is a (signed) measure $\mu$ on $G$ such that

$$
\begin{aligned}
& \operatorname{Re} \int_{G} p(x) d \mu(x)<K \quad \text { for } \quad p \in U, \\
& \operatorname{Re} \int_{G} p(x) d \mu(x) \geq K \quad \text { for } \quad p \in W .
\end{aligned}
$$


We show that the measure $\nu$ defined by $\nu(A)=\mu(-A)$ has the desired properties. First, if $\gamma \in E$, then $K \gamma \in W$, hence we see that

$$
K \leq \operatorname{Re} \int_{G} K \gamma d \mu=K \operatorname{Re} \hat{\mu}(-\gamma)
$$

i.e., $|\hat{\nu}(\gamma)| \geq 1$. Second, if $\gamma \notin E$ and $\varepsilon$ is any complex number, then $p=K \varepsilon \gamma \in U$ (because $\hat{p}(\lambda)=0$ for $\lambda \in E)$. Thus, $K>\operatorname{Re} K(\varepsilon \hat{\nu}(\gamma)$ ), and $\hat{\nu}(\gamma)=0$.

Now we can restate Theorem 16 as follows: if $E$ is a quasi-Marcinkiewicz set but not a quasi-Cohen set, then $C_{E}(G)$ fails to have G-L l.u.st.

18. Sidon sets and arithmetic diameter. For a finite set $\Lambda \subset \Gamma$, its arithmetic diameter $d(\Lambda)$ is defined as the smallest $N$ such that $C_{\Lambda}(G)$ is at most 2-distant (relative to the Banach-Mazur distance) from a subspace of the $N$-dimensional space $l_{N}^{\infty}$. If $E$ is a Sidon set in $\Gamma$, then $C_{\Lambda}(G)$ is at most $S(E)$-distant from $l_{|\Lambda|}^{1}$ for every finite $\Lambda \subset E$. Consequently, the arithmetic diameter of $\Lambda$ must grow exponentially as a function of $|\Lambda|$.

There are many ways to see this. For instance, we may argue as follows. First, recall that for $p \geq 2$ the type 2 constant of $L^{p}$ is of order $c \sqrt{p}$. Next, it is easily seen that the Banach-Mazur distance between $l_{N}^{\infty}$ and $l_{N}^{\log N}$ is bounded uniformly in $N$, so the type 2 constant of $l_{N}^{\infty}$ does not exceed $c \sqrt{\log N}$. Finally, if $e_{1}, \ldots, e_{n}$ are the coordinate unit vectors in $l_{n}^{1}$, then the Rademacher average $\int_{0}^{1}\left\|\sum r_{i}(t) e_{i}\right\| d t$ is equal to $n$, so that the type 2 constant of $l_{n}^{1}$ is at least $\sqrt{n}$. Thus, if $l_{n}^{1}$ is 2-embeddable in $l_{N}^{\infty}$, then $\sqrt{n} \leq C^{\prime} \sqrt{\log N}$, as desired.

It is remarkable that the exponential dependence of $d(\Lambda)$ on $|\Lambda|$ in fact characterizes the Sidon sets.

Theorem 20 (Bourgain [Bo1]). If $\log d(\Lambda) \geq \delta|\Lambda|$ for every finite $\Lambda \subset E$, then $E$ is a Sidon set. Moreover, we have $S(E) \geq c \delta^{-11}$, where $c$ is a universal constant.

The proof of this Banach-geometric characterization of Sidon sets can hardly be called "geometric"; rather, it is combinatorial, because the only geometric notion involved is that of entropy. In general, in a metric space with metric $\rho$, the $\varepsilon$ entropy of a set $F$ is the logarithm of the smallest cardinality $N_{\rho}(\varepsilon)=N_{\rho}(F, \varepsilon)$ of an $\varepsilon$-net for $F$.

The idea of using entropy in the theory of Sidon sets was originally exploited by Pisier (see, e.g., [Pi2]). Later, Bourgain revised Pisier's work on Sidon sets, replacing some fine probability methods by an elementary random choice combined with entropy combinatorics and with harmonic analysis arguments. We refer the reader to the Bourgain's survey [Bo1] (and to the references therein) for this. The following statement is [Bo1, Corollary 8]; this is a slight improvement of Pisier's original entropy characterization of Sidon sets (see [Pi2, p.941]). For a subset $\Lambda$ of $\Gamma$, we define a metric $\rho^{\Lambda}$ on $G$ as follows:

$$
\rho^{\Lambda}(x, y)=\sup _{\gamma \in \Lambda}|\gamma(x)-\gamma(y)|
$$

Theorem 21. Let $E \subset \Gamma$. If for every finite set $\Lambda \subset E$ and some $\tau>0$ we have

$$
N_{\rho^{\Lambda}}(G, \tau)>2^{\sigma|\Lambda|},
$$


then $E$ is a Sidon set and $S(E) \leq C \sigma^{-10}\left(\log \frac{2}{\tau}\right)^{10}$.

We show how Theorem 20 is deduced from Theorem 21. Suppose that $E \subset \Gamma$ is such that $\log d(\Lambda) \geq \delta|\Lambda|$ for all finite sets $\Lambda \subset E$. For any finite $\Lambda$, along with $\rho^{\Lambda}$ we introduce another (greater) metric $\varkappa^{\Lambda}$ on $G$ :

$$
\varkappa^{\Lambda}(x, y)=\sup \left\{|f(x)-f(y)|: f \in C_{\Lambda}(G),\|f\|_{\infty} \leq 1\right\} .
$$

It is easily seen that for the arithmetic diameter $d(\Lambda)$ we have $d(\Lambda) \leq N_{\varkappa^{\Lambda}}(G, 1 / 3)$. Indeed, denoting the latter quantity by $N$, we find a $(1 / 3)$-net $x_{1}, \ldots, x_{N}$ for $G$ in the metric $\varkappa^{\Lambda}$. Then the mapping $f \mapsto\left(f\left(x_{1}\right), \ldots, f\left(x_{N}\right)\right)$ is a good embedding of $C_{\Lambda}(G)$ into $l_{N}^{\infty}$.

Next, fixing a finite set $\Lambda \subset E$, we clearly have

$$
\varkappa^{B}(x, y) \leq S(\Lambda) \rho^{B}(x, y)
$$

for all $B \subset \Lambda$, yielding

$$
N_{\rho^{B}}\left(G, \frac{1}{3 S(\Lambda)}\right) \geq N_{\varkappa^{B}}\left(G, \frac{1}{3}\right) \geq d(B) \geq 2^{\delta|B|} .
$$

Now, Theorem 21 implies the inequality

$$
S(\Lambda) \leq C \delta^{-10} \log (6 S(\Lambda))
$$

which does not allow the quantity $S(\Lambda)$ to grow infinitely as $\Lambda$ expands. More precicely, we obtain $S(\Lambda) \leq C^{\prime} \delta^{-11}$ for every finite $\Lambda \subset E$, whence $S(E) \leq C^{\prime} \delta^{-11}$.

\section{Sidon sets and cotype.}

Theorem 22 (Bourgain and Milman; see [BoMi, Bo1]). If $C_{\Lambda}(G)$ is of cotype $q$ for some $q<\infty$, then $\Lambda$ is a Sidon set.

Conversely, if $\Lambda$ is a Sidon set, then $C_{\Lambda}(G) \sim l^{1}(\Lambda)$, which is of cotype 2.

Recalling the well-known characterization of the spaces with finite cotype, we can restate Theorem 22 in the following way: either $\Lambda$ is a Sidon set, or $C_{\Lambda}$ contains the spaces $l_{n}^{\infty}$ uniformly.

Theorem 22 can be deduced from Theorem 20 and the following subtle fact of the theory of finite-dimensional Banach spaces.

Lemma 23. Let $X$ be an n-dimensional normed space 2-isomorphic to a subspace of $l_{N}^{\infty}$. Then $n \leq C\left[C_{q}(X) \log C_{2}(X)\right]^{q} \log N$.

Here $C_{r}(X), 2 \leq r<\infty$, is the cotype $r$ constant of $X$.

We refer to $[\mathrm{Bo} 1]$ for the proof of this lemma.

Now, we prove Theorem 22. Let $E \subset \Gamma$, and let $C_{E}(\Gamma)$ be of cotype q, i.e., $C_{q}\left(C_{E}(G)\right)<\infty$. We fix a finite set $\Lambda \subset E$ and obtain an a priori estimate for $S(\Lambda)$ (like that at the end of the preceding section). If $B \subset \Lambda$, then $C_{2}\left(C_{B}(G)\right) \leq S(\Lambda)$. By Lemma 23

$$
|B| \leq C\left[C_{q}\left(C_{E}(G)\right)\right]^{q}[\log S(\Lambda)]^{q} \log d(B),
$$

where, as before, $d(B)$ stands for the arithmetic diameter of $B$. Now, Theorem 20 implies that

$$
S(\Lambda) \leq C^{\prime}\left[C_{q}\left(C_{E}(G)\right) \log S(\Lambda)\right]^{11 q},
$$


which does not allow the numbers $S(\Lambda)$ to be unbounded. Thus, $E$ is a Sidon set.

It should be mentioned that the case of $q=2$ in Theorem 22 is less involved (this case had been analyzed independently by Pisier and by Kwapień and Pełczyński prior to Theorem 22). We can argue nearly as in the proof of Theorem 18. Indeed, let $C_{E}(G)$ be of cotype 2. For a function $x \in C_{E}(G)$, the operator $\tau$ of convolution with $x(-t)$ can be viewed, in a natural way, as a mapping from $C_{E}(G)^{*}$ to $C_{-E}(G)$. So, the domain of $\tau$ is the conjugate of a cotype 2 space, and its range is a cotype 2 space. By Pisier's factorization theorem (see [Pi3, Chapter 3]), $\tau$ factors through a Hilbert space $H$. This factorization $\tau=\beta \alpha$ is shown in the following diagram:

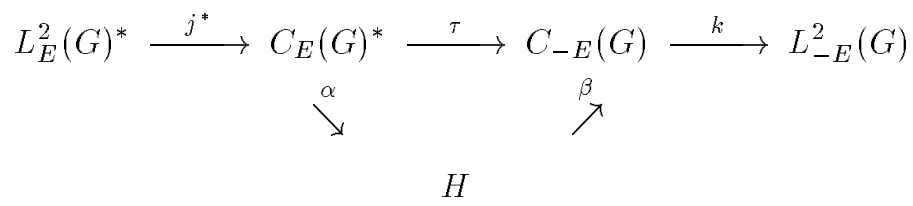

Here $j$ and $k$ are identity embeddings. Now, $k$ is 1 -summing, hence $k \beta$ is HilbertSchmidt. Considering the adjoints, in a similar way we see that $\alpha j^{*}$ is HilbertSchmidt. Concequently, the composition $k \beta \alpha j^{*}$ is nuclear with $\nu_{1}\left(k \beta \alpha j^{*}\right) \leq C\|x\|_{\infty}$ Since this composition is again the operator of convolution with $x(-t)$, we obtain $\sum_{\gamma \in E}|\hat{x}(\gamma)| \leq C\|x\|$.

20. Multipliers on spaces of vector-valued functions. Let $X$ be a Banach space; then the spaces of $X$-valued functions $L^{p}(G ; X)(1 \leq p \leq \infty)$ or $C(G, X)$ are defined as usual. As in the scalar case, for $E \subset \Gamma$ the spaces $L_{E}^{p}(G ; X)$ and $C_{E}(G ; X)$ are distinguished by the condition

$$
\hat{f}(\gamma) \stackrel{\text { def }}{=} \int_{G} f \bar{\gamma}=0 \quad \text { for } \quad \gamma \notin E .
$$

Now, assume we are given a bounded multiplier of scalar spaces $T_{m}: L_{E_{1}}^{p_{1}}(G) \rightarrow$ $L_{E_{2}}^{p_{2}}(G)$, acting in accordance with formula (1). The expression $m \cdot \hat{f}$ makes sense also for $X$-valued functions $f$, so it is natural to ask about the description of the spaces $X$ for which (1) generates a bounded operator from $L_{E_{1}}^{p_{1}}(G, X)$ to $L_{E_{2}}^{p_{2}}(G, X)$.

In fact, for each particular multiplier $T_{m}$ this question presents a mystery, which can be clarified only rarely, and by dissimilar techniques. Below we briefly discuss two important cases. Beyond this, we mention the paper [BIPe], in which the vectorvalued analogs of the Paley inequality (see Theorem 3) and the Hardy inequality

$$
\sum_{n \geq 0}(n+1)^{-1}|\hat{f}(n)| \leq C\|f\|_{1}, \quad f \in H^{1}(\mathbb{T}),
$$

as well as some related questions were discussed. In spite of a quite considerable bulk of information presented in [BIPe], no complete description of the corresponding classes of Banach spaces $X$ is available in these cases as yet.

21. B-convexity and $K$-convexity. Here our basic multiplier is the orthogonal projection of $L^{2}(D)$ ( $D$ is the dyadic group, see Section 8 for the definition) onto the subspace generated by the coordinate functions of $D$. (Identifying $D$ with $[0,1]$ in the usual way, we arrive at the Rademacher projection.) The spaces $X$ for which this projection extends in a natural way to $L^{2}(D ; X)$ are said to be $K$-convex.

The notion of $K$-convexity is quite useful in the theory of Banach spaces, primarily due to the fact that it is intimately related to the duality between type and cotype. Remarkably, $K$-convex spaces admit a complete characterization. 
Theorem 23 (Pisier; see [Pi4]). A Banach space $X$ is $K$-convex if and only if $X$ does not contain the spaces $l_{n}^{1}$ uniformly.

The spaces with the latter property are called $B$-convex. A space is $B$-convex if and only if it is of nontrivial type; see "Basic Concepts".

22. UMD-spaces. Here our basic multiplier is the Hilbert transformation $\mathcal{H}$ on $L^{2}(\mathbb{T})$. We have $\mathcal{H}=T_{m}$, where $m(k)=-i \operatorname{sgn} k, k \in \mathbb{Z}$.

It is really quite useful to know for which Banach spaces $X$ the Hilbert transformation (more generally, an arbitrary Calderón-Zygmund singular integral operator) acts on the $L^{p}$-space of $X$-valued functions. The general theory reduces the case of any $p \in(1, \infty)$ to the case of $p=2$; see, e.g., [St].

Surprisingly, the class of spaces $X$ for which $\mathcal{H}$ acts on $L^{2}(\mathbb{T}, X)$ admits a complete description.

Theorem 24 (Burkholder, McConnel, Bourgain). A Banach space X has the above property if and only if there is a biconvex function $\zeta: X \times X \rightarrow \mathbb{R}$ such that $\zeta(0,0)>0$ and $\zeta(x, y) \leq\|x+y\|$ if $\|x\|=\|y\|=1$.

The same condition is equivalent to the continuity of standard martingale transformations on $L^{2}(X)$. That is why the spaces $X$ such that $\mathcal{H}$ acts on $L^{2}(X)$ are called UMD-spaces (UMD is for "unconditionality of martingale differences"). The proof of Theorem 24 is indirect and passes via this statement on martingales. We refer the reader to Burkholder's survey [Bu] and to the references in it for the details.

It should be noted that the condition formulated in Theorem 24 is difficult to work with. Basically, the only way to prove the existence of the above $\zeta$ on a Banach space $X$ is to verify the continuity of $\mathcal{H}$ (or of the martingale transformations) on $L^{2}(X)$ directly. The Hilbert space seems to be the only one presenting a simple possibility of exhibiting $\zeta$ (for instance, we may put $\zeta(x, y)=1+\operatorname{Re}\langle x, y\rangle$ ).

Direct verification of the continuity of $\mathcal{H}$ shows that the spaces $L^{p}$ with $1<p<$ $\infty$ and the Shatten-von Neuman classes $C_{p}$ (again with $1<p<\infty$ ) are UMDspaces. Next, the property of being a UMD-space is inherited by the subspaces and quotient spaces. This is a superproperty (if $X$ is finitely representable in $Y$ and $Y$ is UMD, then so is $X$ ). A UMD-space must be superreflexive, but not all superreflexive spaces are UMD. As before, we refer the reader to [Bu] and the references therein. Again, the proofs of the above statements do not use Theorem 24.

23. The above discussion should be supplemented with the following result due to Bourgain.

Theorem 25. If $\mu$ is a finite measure and $0<r<1$, then the Hilbert transformation $\mathcal{H}$ is bounded from $L^{2}\left(\mathbb{T} ; L^{1}(\mu)\right)$ to $L^{2}\left(\mathbb{T} ; L^{r}(\mu)\right)$.

We refer the reader to the survey [Ki1] for the proof and related material. The result can be used to verify statement (ii) in Section 8 . This idea is Bourgain's; the details of this verification can also be found in [Ki1].

24. Returning once again to statements (i)-(v) in Section 8 , we note that we may ask any Banach space theory question about any specific space arising in harmonic analysis. This will yield an incontestable point of contact of the two fields, but rarely will this show a real interplay between them. In many cases, a pure problem 
of hard analysis (even without the adjective "harmonic") arises in this way, as is described in Section 8 after statements (i) $-(\mathrm{v})$.

Some exceptions of these "rule" were, however, discussed above. Another one is presented by the still mysterious space $U$ of uniformly convergent Fourior series on the unit circle. Bourgain was the first to prove that $U^{*}$ is weakly sequentially conplete, and his proof involved difficult techniques of hard analysis; see [Bo3]. But later it was discovered that the statement can be verified almost entirely within the ("soft") methods of functional analysis. We refer the reader to the paper [GaKi] in this collection for a discussion and references.

Having mentioned the space $U$, we probably cannot avoid considering the spaces of trigonometric polynomials on the circle. Let

$$
\mathcal{P}_{n}^{p}=\operatorname{span}\left\{1, z, \ldots, z^{n}\right\}
$$

with the metric of $L^{p}(\mathbb{T}), 1 \leq p \leq \infty$. In a way (and from the harmonic analysis viewpoint), these spaces may be regarded as "elementary $(n+1)$-dimensional blocks" building the Hardy classes $H^{p}(\mathbb{T})$. For $1<p<\infty$ this is emphasized by the fact that the $\mathcal{P}_{n}^{p}$ are complemented in $H^{p}$ uniformly in $n$. However, for $p=1$ or $p=\infty$ the norm of the invariant projection of $H^{p}$ onto $\mathcal{P}_{n}^{p}$ grows as $c \log n$ (and, by averaging, the norm of any other projection cannot be smaller). The following observation (folklore) was made by Bourgain and Pełczyński about 20 years ago.

Proposition 26. The spaces $\mathcal{P}_{n}^{1}$ (respectively, $\mathcal{P}_{n}^{\infty}$ ) can be embedded uniformly complementedly in $H^{1}$ (respectively, $H^{\infty}$ ).

Proof. We treat only the case of $H^{1}$, the other one being similar. Considering the subspaces generated by the odd or by the even powers of $z$, it is easy to deduce that $H^{1} \sim H^{1} \oplus H^{1}$. Thus, instead of $H^{1}$, we embed $\mathcal{P}_{n}^{1}$ into $\bar{z} \bar{H}^{1} \oplus H^{1}=$ $L_{\mathbb{Z}_{-}}^{1}(\mathbb{T}) \oplus L_{\mathbb{Z}_{+}}^{1}(\mathbb{T})$. The embedding in question is given by the formula

$$
I_{n}: p \mapsto\left(\bar{z}^{n+1} p, p\right), \quad p \in \mathcal{P}_{n}^{1}
$$

Now, we define an operator $J_{n}: L_{\mathbb{Z}_{-}}^{1} \oplus L_{\mathbb{Z}_{+}}^{1} \rightarrow \mathcal{P}_{n}^{1}$ by the formula

$$
J_{n}(f, g)=z^{n+1} K_{n} * f+K_{n} * g,
$$

where $K_{n}$ is the $n$th Fejér kernel. It is easily seen that $J_{n} I_{n}=\mathrm{id}_{\mathcal{P}_{n}^{1}}$.

Without entering into the details, we refer the reader to [Bo4] for a construction of a good basis in $\mathcal{P}_{n}^{\infty}$, and to [GoR] for evaluation of various constants (such as G-L l.u.st., Banach-Mazur distances to various spaces, etc.) for the spaces $\mathcal{P}_{n}^{p}$.

Let us stop at this point.

\section{REFERENCES}

[AM] Alspach D. E., Matheson A., Projections onto translation-invariant subspaces of $L_{1}(\mathbb{R})$, Trans. Amer. Math. Soc. 277 (1983), no. 2, 815-823.

[BE] Bachelis G. F., Ebenstein S. E., On $\Lambda(p)$ sets, Pacif. J. Math., 54 (1974), no. 1, 35-38.

[BIPe] Blasco O., Pelczyński A., Theorems of Hardy and Paley for vector-valued analytic functions and related classes of Banach spaces, Trans. Amer. Math. Soc. 323 (1991), no. $1,335-367$. 
[Bo1] Bourgain J., Subspaces of $L_{N}^{\infty}$, arithmetical diameter, and Sidon sets. In: Probability in Banach spaces, V. Medford, Mass, 1984. Lecture Notes in Mathematics, 1153. Springer, Berlin.

[Bo2] Bourgain J., Bounded orthogonal sets and the $\Lambda(p)$ set problem, Acta Math. 162 (1989), $227-246$.

[Bo3] Bourgain J., Quelques propriétés linéaires topologiques de l'espace des séries de Fourier uniformement convergentes, Séminaire Initiation d'Analyse (G. Choquet, M. Rogalski, J. Saint Raymond, Eds.), 22e année, Exposé no. 14, Univ. Paris-6, 1982-83.

[Bo4] Bourgain J., Homogeneous polynomials on the ball and polynomial bases, Isr. J. Math. 68 (1989), no. 3, 327-347.

[BoMi] Bourgain J., Milman V., Dichotomie du cotype pour les espaces invariants, C.R. Acad. Sc. Paris, 300 Sér I (1985), no. 9, 263-266.

[Bu] Burkholder D. L., Martingales and Fourier analysis in Banach spaces, Lecture Notes Math., 1206, 61-108, Springer, Berlin etc., 1986.

[DJT] Diestel J., Jarchow H., Tonge A., Absolutely Summing Operators, Cambridge University Press, 1995.

[GaKi] Gamelin T. W., Kislyakov S. V., Uniform algebras as Banach spaces, this collection.

[GoR] Gordon Y., Reisner S., Some geometrical properties of Banach spaces of polynomials, Isr. J. Math. 42 (1982), no. 1-2, 99-116.

[Gr-McG] Graham C. C., McGehee O. C., Essays in commutative harmonic analysis, Springer, Berlin, 1979.

[Gre] Greenleaf F. P., Invariant means on topological groups and their applications, Van Nostrand-Keinhold Company, New York etc., 1969.

[Gu] Gundy R. F., Inégalités pour martingales à un et deux indices, Lecture Notes Math. 774 (1980), 251-334.

[H] Hoffman K., Banach spaces of analytic functions, Prentice Hall, Englewood Cliffs, N.Y., 1962.

[KaS] Kahane J.-P., Salem R., Ensembles parfaits et séries trigonométriques, Hermann, Paris, 1963.

[KadPe] Kadec M.I., Pelczyński A., Bases, lacunary sequences and complemented subspaces in the spaces $L^{p}$. Studia Math. 21 (1961/62), 161-176.

[KalPe] Kalton N. J., Pelczyński A., Kernels of surjections from $\mathcal{L}_{1}$-spaces with an application to Sidon sets, Math. Ann. 309 (1997), no. 1, 135-158.

[Ki1] Kisliakov S. V., Absolutely summing operators on the disk algebra, St. Petersburg Math. J. 3 (1992), no. 4.

[Ki2] Kisliakov S. V., Some more Banach spaces satisfying Grothendieck's theorem, St. Petersburg Math. J. 7 (1996), no. 1.

[Kl] Klemes I., Idempotent multipliers of $H^{1}$, Canad. J. Math. 39 (1987), no. 5, 1223-1234.

[KwPe] Kwapień S., Pelczyński A., Absolutely summing operators and translation invariant spaces of functions on compact abelian groups, Math. Nachr. 94 (1980), 303-340.

[LoR] Lopez J. M., Ross K. A., Sidon sets. Lecture notes in pure and appl. math., 13, M. Dekker, New York, 1975.

[Ma] Maurey B., Théorèmes de factorization pour les opérateurs linéaires à valeurs dans les espaces $L^{p}$, Astérisque 11 (1974).

[MiSch] Milman V. D., Schechtman G., Asymptotic theory of finite dimensional normed spaces, Lecture notes Math., 1200, Springer-Verlag, Berlin etc., 1986.

[Pe1] Pełczyński A., p-integral operators commuting with group representations and examples of quasi p-integral operators which are not p-integral, Studia Math. 33 (1969), 63-70.

[Pe2] Pelczynśki A., Linear extensions, linear averagings, and their applications to linear topological classification of spaces of continuous functions, Dissertationes Mathematicae (Rozprawy matematyczne), LVIII, PWN, Warszawa, 1968.

[Pi1] Pisier G., De nouvelles caractérisations des ensembles de Sidon, Advances in Math., Supplementary studies, Math. Analysis and Appl. (part B) 7 (L. Nachbin, editor) (1981).

[Pi2] Pisier G., Conditions d'entropie et caractérisations arithmétiques des ensembles de Sidon, Topics in Modern Harmonic Analysis, Proc. of a Seminar held in Torino and Milano, May-June 1982, vol.II. Instituto Nazionale di Alta Matematica Francesco Severy, Roma, 1983. 
[Pi3] Pisier G., Factrization of linear operators and geometry of Banach spaces, Reg. Conf. Ser. in Math. no. 60, Amer. Math. Soc., Providence, R.I. (1986).

[Pi4] Pisier G., Holomorphic semi-groups and the geometry of Banach spaces, Annals of Math. 115 (1982), 375-392.

[Ro] Rosenthal H.P., Projections onto translation-invariant subspaces of $L^{p}(G)$, Mem. Amer. Math. Soc. 63 (1966).

[Ru] Rudin W., Trigonometric series with gaps, Math. Mech. 9 (1960), 203-227.

[St] Stein E. M., Singular integrals and differentiability properties of functions, Princeton University Press, 1970.

[T] Talagrand M., Sections of smooth convex bodies via majorizing measures, Acta Math. 175 (1995), no. 2, 273-300.

[X] Xu Q., Some properties of the quotient space $L^{1}\left(\mathbb{T}^{n}\right) / H^{1}\left(\mathbb{D}^{n}\right)$, Illinois J. Math. 37 (1993), no. 3, 437-454.

[Z] Zygmund A., Trigonometric series, v. I-II, Cambridge University Press, 1959. 